Aus dem Sanatorium Deutselies Haus Agra, Niederlassung der Deutschen Heilstätte in Davos (Chefarzt: Geh. Sanitätsrat Dr. K alle).

\title{
Pneumothorax artificialis und Pleuritis exsudativa der Gegenseite.
}

Von

Dr. H. Süssdorf, Assistenzarzt.

Wäbrend das Auftreten eines Exsudates im Pneumothoraxraum eine allen mit der Pneumothoraxbehandlung der Lungentuberkulose vertrauten Ärzten wohl bekannte Erscheinung ist (zeigt sich ein solches doch nach L. Spengler u. a. in $50 \%$ aller Pneumothoraxfälle), darf eine Pleuritis exsudativa auf der gesunden Seite zu den grössten Seltenheiten gerechnet werden, so dass sogar Brauer und Spengler in ihrer Abhandlung über die Lungenkollapstherapie im SchröderBlumenfeldschen Handbuch über keinen einschlägigen Fall weder aus persönlicher Beobachtung noch auch nur referierend aus der Literatur berichten konnten. Es mag deshalb ein in letzter Zeit hier zur Behandlung gekommener Fall von mehr als nur spezialistischem Interesse sein, da mit dem Fortschreiten der Pneumothoraxtechnik immer weitere Ärztekreise sich des Verfahrens bedienen, und dabei oftmals eine gewisse Unsicherheit des therapeutischen Handelns einzutreten scheint angesichts der speziell durch Exsudatbildung bedingten Zwischenfälle, eine Wahrnehmung, die wir mehrmals in letzter Zeit zu machen Gelegenheit hatten. Bisher hatten aber auch wir hier unter unsern nunmehr über 140 Pneumothoraxpatienten wohl gelegentlich Aktivierungserscheinungen in der gegenseitigen Lunge, nie aber exsudative Prozesse der Pleura daselbst beobachtet.

Es handelte sich in unserem Fall um eine 32jährige Frau, aus deren Krankengeschichte folgende Daten hervorzuheben wären: in familia keine Tuberkulose, sie selbst immer gesund (3 normale Geburten, deren letzte 1918), bis sie ab Juli 1918 unter Müdigkeit zu leiden hatte und eine im Oktober 18 aufgetretene Grippeerkrankung die bisher wohl latente Lungentuberkulose zum Aufflammen brachte. In der nächsten Zeit mehrmals Blutungen, während deren sie meist vorübergehend und schliesslich von Dezember 18 ab dauernd mit Fieber zu Bett lag. Es wurde ihr deshalb im Dezember 18 von ihrem Arzt ein linksseitiger Pneumothorax angelegt, der aber zunächst keine 
Besserung, auch keinen Abfall der Temperaturen brachte. Im Gegenteil diese stiegen noch höher, nicht zuletzt wohl unter dem Einfluss eines im April 19 aufgetretenen Exsudats im Pneumothoraxraum. Schliesslich kam Patientin am 25. VII. 1919 in Agra zur Aufnahme.

Der Aufnahmestatus ergab einen completten linksseitigen Pneumothorax mit Exsudat bis zur Höhe der 4. Rippe bzw. oberhalb Angulus, während die rechte Seite klinisch und röntgenologisch frei war bis auf die bei Pneumothorax übliche starke Strangzeichnung und einzelne kleine knotenförmige Verschattangen im Hilusgebiet. Eine Probepunktion des Exsudats im Pneumothoraxraum ergab klar seröses Exsudat, dessen mikroskopische Untersuchung spärlich Lymphozyten und vereinzelte Tuberkelbazillen. Sputum $70 \mathrm{ccm}$, Tbc. negativ; Temperatur bis 38,7, rektal. Allgemeinbefinden sehr schlecht. Nach längerer Beobachtung ohne Befundsänderung wurden am 5. VIII. $150 \mathrm{ccm}$ und am 25. VIII. weitere $500 \mathrm{ccm}$ klar seröser Flüssigkeit abpunktiert (entgegen unserem sonstigen Verfahren ohne Stickstoffersatz, da die stark komprimierte Lunge leichte Verdrängungserscheinungen seitens des Herzens verursachte). Danach sinkt unter kleinen Pyramidongaben die Temperatur langsam bis Ende September zur Norm bei gleichzeitigem allmählichen Wiederansteigen des Exsudates, dessen Spiegel schliesslich am 20. IX. 19 wieder in Höhe der 3. Rippe bzw. Mitte Scapulae liegt. Die Temperaturen hielten sich danach auch ohne Antipyretica normal unter fortschreitender Besserung des Allgemeinbefindens, so dass Patientin im Oktober das Bett verlassen konnte, bis zum 20. XII. 19. Da liess die Durchleuchtung eine Neigung des linken Unterlappens zur Entfaltung unter dem Flüssigkeitsspiegel vermuten, weshalb erneute Punktion von im ganzen $500 \mathrm{ccm}$ seröser Flüssigkeit und Nachfüllung von $500 \mathrm{ccm} N$. bis .zum Enddruck $-4-2$ (Wasser), wonach röntgenologisch der Unterlappen wieder gute Kompressionsverhältnisse zeigt bei nur noch geringer Exsudatspur im Sinus phrenico-costalis.

24. XII. 19. Nach Punktion und Nachfüllung vor 4 'Tagen Temperaturanstieg bis 38,6, bis heute langsamer Abfall zur Norm. Patientin fäbrt danach trotz Abraten unsererseits zur Weihnachtsfeier in ihre ca. $10 \mathrm{~km}$ entfernte Wohnung.

2. I. 20. Wiederaufnahme im Sanatorium Agra. Das gute Befinden hatte die ersten Tage auch zu Hause angehalten bis am 29. XII. plötzlich unter starken Schmerzen in der rechten Brustseite die Temperatur über $\mathbf{3 9}$ stieg. Befinden in den nächsten 'lagen unverändert bis zur Wiederaufnahme hier mit folgendem Lungenstatus: R. v. o. verschärftes Atmen ohne Nebengeräusche, r. v. u. seitlich leises Knarren; r. b. o. verschärftes Atmen ab Mitte Scapulae leises Knarren, das nach unten $\mathrm{zu}$ weniger deutlich wird; daselbst $r$. h. u. etwas verkürzter Schall, abgeschwächtes Atmen ohne deutliche Nebengeräusche. L. v. stark abgeschwächtes Atmen ohne Nebengeräusche, desgleichen 1. h.; Exsudat perkutorisch nicht sicher feststellbar. Röntgenologisch sind die Pneumothoraxverhältnisse gut bei geringem Sinusexsudat; $r$. $u$. ist eine unbedeutende Verschattung zu sehen. Temperatur 38-39,0 bei starken Schmerzen in rechter Brustseite; Allgemeinbefinden schlecht, Diurese mangelhaft. 
6. I. Probepunktion rechts ergibt $15 \mathrm{ccm}$ klar seröser Flïssigkeit (mikroskopisch spärlich Lymphozyten, steril); Betinden unverändert.

13. I. Die Durchleuchtung zeigt nunmehr r. diffuse Verschattung bis in die Hilusgegend, Pneumothorax links unverändert. Erneute Punktion von $50 \mathrm{ccm}$ nunmehr hämorrhagischer Flüssigkeit.

20. I. Im Anschluss an die letzte Punktion fingen die Temperaturen unter Brustwickeln mit Senfmehl bzw. Schmierseife sowie kleinen kombinierten Pyramidon- bzw. Aspirin- Chiningaben langsam an, abzufallen unter Resorption des Exsudats, die sich auch in gesteigerter Diurese kundgibt, so dass heute vor dem Röntgenschirm eine wesentliche Aufhellung der rechtsseitigen unteren Lungenpartien festzustellen ist.

15. II. Temperatur nunmehr auch ohne Fiebermittel normal. Perkutorisch deutliche Aufhellung der rechtsseitigen Partieen.

20. II. Pneumothoraxlunge beginnt sich langsam zu entfalten, deshalb Nachfüllung von $270 \mathrm{ccm}$ N. zu einem Enddruck von $-1-4$ (Anfangsdruck $-14-13$ ).

25. II. Nach Nachfüllung leichte Temperatursteigerung ohne sonstige Beschwerden, die bis gestern aber wieder zurückgegangen war. Heute wieder gutes Allgemeinbefinden. Patientin beginnt aufzustehen.

20. III. Dauernd gutes Allgemeinbefinden. Patientin ist ausser Bett. Gestern erneute kleine Nachfüllung von $250 \mathrm{~N}$. ohne Folgeerscheinungen. Status praesens: R.v. o. verschärftes Atmen ohne Nebengeräusche, r. v. u. seitlich leichte Schallierkürzung, Atmung etwas schwächer, keine Nebengeräusche; r.h.o, verschärftes Atmen ohne Nebengeräusche, neben Spina Knarren, undeutlich auch unter Spina, weiter abwärts schwächeres Atmen rauh ohne Nebengeräusche; $r . h$. $u$. ab Angulus verkürzter Schall mit abgeschwächtem Atmen ohne Nebengeräusche. L. y. und 1. h. stark abgeschwächtes Atmen, keine Nebengeräusche; l.u. seitlich schmale Dämpfungszone (beweglich). Röntgenologisch r. u. leichte Sinusverschattung, l. u. den Șinus ausfüllendes bewegliches Exsudat.

Im ganzen also ein glatt verlaufender Fall einer Pleuritis exsudativa dextra bei gleichzeitig bestehendem Pneumothorax artificialis der linken Seite, und zwar genau 12 Monate nach dessen Anlegung. Inwieweit zur Auslösung des Zwischenfalles die kurz nach der Punktion und Nachfüllung des linksseitigen Pneumothorax bewerkstelligte Übersiedlung der Patientin nach Hause mit der veränderten Lebensweise daselbst beigetragen hat, ist natürlich schwer festznstellen. Immerhin ist zu beachten, dass die sog. gesunde Seite bei längerer Beobachtung hier sowohl klinisch als röntgenologisch wirklich gesund schien. Die therapeutischen Massnahmen beschränkten wir auf die bei Pleuritis exsudativa üblichen kleinen Punktionen (irgend welche Erscheinungen einer Zirkulationsstörung oder nennenswerte Dyspnoe bestanden nicht!) etwa von der 2. Krankheitswoche ab. Sie führten im Verein mit hydrotherapeutischen Massnahmen zu einem vollen Erfolg. Bemerkenswert war das Verhalten des andersseitigen Pneumothorax mit Exsudat, der sich während der langen Wochen absolut ruhig verhielt ohne jeden Eingriff; natürlich war indessen eine beträchtliche Druckverminderung im Pneumothoraxraum entstanden bei allerdings nur geringer Entfaltung der Lunge. Letztere Tatsache bätte aber auch recht verhängnisvoll 
werden können, wenn stärkere Atmungsbehinderung die Mitarbeit der Pneumothoraxlunge erfordert hätte. Wir vermeiden deshalb für gewöhnlich bei unsern Pneumothoraxpatienten jede stärkere Druckanwendung, indem wir unter nur mässiger Kompression lediglich eine Ruhigstellung der erkrankten Lunge erstreben. Im vorliegenden Fall hatte jedoch das Exsudat eine starke Kompression bedingt und so entsprechende Schrumpfung von Lunge und Pleura pulmonalis verursacht, wie diē exsudativ-pleuritischen Prozesse andererseits auch die Resorptionsfähigkeit der Pleura herabgesetzt haben. Interessant war uns endlich die Beobachtung des nach der Punktion vor der Pleuritis gebliebenen kleinen Exsudatrestes. Während früher nach Punktionen das Exsudat sich meist sehr rasch wieder ersetzte, blieb es diesmal im wesentlichen unverändert.

Für die Praxis ergeben sich so therapeutisch die Richtlinien, dass auch solche komplizierende exsudativ-pleuritische Prozesse allgemein nach den für die Behandlung der Pleuritis exsudativa bzw. des Pneumothorax mit Exsudat üblichen Regeln angegangen werden durch wiederholte kleinere Punktionen und ableitende Massnahmen der akut erkrankten Pleura unter möglichst konservativer Behandlung des Pneumothorax event. durch nur kleine den 0-Druck eben herstellende Nachfüllungen. Natürlich hätten Erscheinungen von Kreislauf- und Atmungsbehinderung entsprechend energischeres Eingreifen verlangt, wie auch die Möglichkeit derartiger schwererer Komplikationsfolgen wieder zur Vorsicht mahnt vor absichtlich forcierter Kompression der Pneumothoraxlunge.

Anmerkung bei der Korrektur: Kurz vor Eingang des Korrekturabzugs erhielt ich Kenntnis durch Liebes Referat (Münch. med. Wochensctrr. 20, $\mathrm{Nr}$. 16) von einem ähnlichen von Als-Faxinge-Sanatorium in der Zeitschrift für 'Tuberkulose (Bd. 31, Het't 6) veröffentlichten Fall, der offenbar die oben angedeuteten bedrohlichen Erscheinungen zeigte, aber ebenfalls $z a$ einem günstigen Abschluss kam. 